\title{
Screening for minimal hepatic encephalopathy in patients with cirrhosis by cirrhosis-related symptoms and a history of overt hepatic encephalopathy
}

\author{
EMI YOSHIMURA ${ }^{1}$, TATSUKI ICHIKAWA ${ }^{2}$, HISAMITSU MIYAAKI ${ }^{1}$, NAOTA TAURA ${ }^{1}$, SATOSHI MIUMA ${ }^{1}$, \\ HIDATAKA SHIBATA $^{1}$, TAKUYA HONDA ${ }^{1}$, FUMINAO TAKESHIMA ${ }^{1}$ and KAZUHIKO NAKAO ${ }^{1}$ \\ ${ }^{1}$ Department of Gastroenterology and Hepatology, Nagasaki University Graduate \\ School of Biomedical Science, Nagasaki 852-8501; ${ }^{2}$ Department of Gastroenterology, \\ Nagasaki Harbor Medical Center, City Hospital, Nagasaki 850-8555, Japan
}

Received March 2, 2016; Accepted May 30, 2016

DOI: $10.3892 /$ br.2016.702

\begin{abstract}
The diagnosis of minimal hepatic encephalopathy (MHE) is more difficult in comparison to the diagnosis of overt hepatic encephalopathy (OHE), as patients with MHE do not exhibit overt neurological deficits and must be diagnosed using specialized equipment. However, identifying MHE is critical for patients with cirrhosis, and a simple screening test is required. The present study aimed to evaluate the associations between MHE, clinical characteristics and questionnaire items regarding sleep disturbances and cirrhosis-related symptom score (CSS). A total of 91 patients who had cirrhosis without $\mathrm{OHE}$ were evaluated using various questionnaires [i.e., CSS, Epworth Sleepiness Scale, the Japanese version of the Pittsburgh Sleep Quality Index (PSQI) and the Japanese 36-item short-form health survey (SF-36)]. MHE was diagnosed using the neuropsychological test. MHE was associated with severe liver damage, which was indicated by liver damage markers and a history of OHE. In addition, MHE was associated with the CSS, PSQI and SF-36 results. The multivariate analyses revealed that a history of OHE was the factor that was the most strongly associated with MHE. Among patients
\end{abstract}

Correspondence to: Dr Tatsuki Ichikawa, Department of Gastroenterology, Nagasaki Harbor Medical Center, City Hospital, 6-39 Shinchi, Nagasaki 850-8555, Japan

E-mail: ichikawa@nagasaki-u.ac.jp

Abbreviations: HE, hepatic encephalopathy; OHE, overt hepatic encephalopathy; NPT, neuropsychological test; MHE, minimal hepatic encephalopathy; PLT, platelets; ALB, albumin; ChE, cholinesterase; MELD, Model for End-stage Liver Disease; CPS, Child-Pugh score; CSS, cirrhosis-related symptom score; ESS, Epworth Sleepiness Scale; PSQI, Pittsburgh Sleep Quality Index; RLS, restless legs syndrome; BCAA, branched-chain amino acid

Key words: overt hepatic encephalopathy history, minimal hepatic encephalopathy, cirrhosis, cirrhosis-related symptom score without a history of OHE, MHE was most strongly associated with CSS, although it was also associated with severe liver damage and platelet counts. A prediction score (calculated using a history of OHE and CSS) provided an area under the receiver operating characteristic curve of 0.738 and a sensitivity of 0.671 for identifying MHE. In conclusion, a history of $\mathrm{OHE}$ and CSS may be useful for identifying MHE in patients with cirrhosis.

\section{Introduction}

Hepatic encephalopathy (HE) is a severe complication among patients with cirrhosis, as it is associated with poor survival outcomes and a reduced quality of life (1). The diagnosis of overt $\mathrm{HE}(\mathrm{OHE})$ is relatively simple, as patients exhibit reduced consciousness and neurological deficits, and treatment can typically be started at the onset of the symptoms. However, minimal HE (MHE) is much more difficult to diagnose, as patients with MHE do not exhibit overt neurological deficits, and sensitive psychometric tests are required to diagnose these patients. The recent guidelines regarding the clinical management of MHE encourage the use of these tests (2), although they require large amounts of time and specialized equipment. For example, the diagnosis of MHE in Japan is typically performed using a personal computer with a specific touch panel (3). Other studies have reported that magnetic resonance spectroscopy (4), neuroelectrical latency (5) and critical flicker frequency (6) can be used to diagnose MHE, although these techniques are also limited by their requirement for specialized equipment. Serum levels of nitrotyrosine (7) and inflammatory cytokines (8) may also be useful for diagnosing MHE, although these techniques are not commercially available in Japan. However, it is critical to diagnose MHE in patients with cirrhosis, as MHE is a risk factor for OHE (9), reduces the 5-year survival rate for cirrhosis (10), impairs the ability of an individual to drive a vehicle (11), increases the incidence of motor vehicle accidents $(12,13)$ and increases the risk of falls (14). Furthermore, MHE is a highly prevalent cognitive disorder that severely affects the health-related quality of life for individuals (15). Additionally, MHE was diagnosed in 
$30.1 \%$ of cirrhotic patients in Japan (16). It shows that MHE is not a rare condition in liver cirrhosis. Therefore, a simple and effective screening method is urgently needed to identify patients with MHE.

Our previous study reported that sleep disturbances in patients with cirrhosis are mainly caused by restless legs syndrome (RLS) (17), and that Child-Pugh scores (CPS) are associated with cirrhosis-related symptom scores (CSS), which are calculated using questionnaire items that were developed in our previous study (18). Furthermore, sleep disturbances and RLS could be accurately diagnosed using this questionnaire and the sleep disturbances in patients without OHE improved following consuming a branched-chain amino acid (BCAA)-enriched snack (18). Moreover, our previous study reported that patients who underwent living donor liver transplantation also experienced sleep disturbances, which were caused by RLS, sleep apnea syndrome and MHE (19). Among these patients, the Japanese 36-item short-form health survey (SF-36) scores were associated with MHE (20), which was diagnosed using a two-dimensional operation system (3). Therefore, the present study aimed to evaluate the associations between MHE, clinical parameters and questionnaire item scores for sleep disturbances, RLS and CSS.

\section{Patients and methods}

Patients. The present study evaluated 91 patients (41 women and 50 men) with cirrhosis who were being evaluated for liver transplantation at Nagasaki University Hospital (Nagasaki, Japan) between July 2011 and May 2014. All the patients had liver cirrhosis, which was diagnosed using laboratory data and imaging findings. None of the patients had OHE at their initial evaluation, and any OHE was subsequently diagnosed using clinical findings.

Diagnosis of MHE. The neuropsychological test (NPT) system is designed to assess psychomotor, attention, memory and special functions in order to diagnose MHE. This system consists of eight tests: Number connection tests A and B, a figure position test, a digit symbol test, a block design test and reaction time tests A-C. The system was simplified to accommodate two-dimensional manipulation using a computer and all tests can be completed in $20 \mathrm{~min}$, which includes the time that is required for practice and reading the operation guide. The software for this system was developed by Otsuka Pharmaceutical Co., Ltd. (Tokyo, Japan), Kokuyo Co., Ltd. (Osaka, Japan) and ISB Co., Ltd. (Tokyo, Japan).

Clinical and laboratory parameters. All the patients underwent anthropometric measurements to calculate body mass index $\left(\mathrm{kg} / \mathrm{m}^{2}\right)$. Laboratory testing was also performed to obtain data regarding the following parameters: White blood cells, red blood cells, platelets (PLT), prothrombin time, blood urea nitrogen, creatinine, aspartate aminotransferase, alanine aminotransferase, $\gamma$-glutamyl transpeptidase, alkaline phosphatase, total bilirubin, total protein, albumin (ALB), high-density lipoprotein, low-density lipoprotein, cholinesterase (ChE), triglycerides, fasting blood glucose and ammonia. Each patient was questioned regarding whether they had a history of OHE, variceal bleeding, ascites or oral
Table I. Clinical characteristics of MHE in patients with cirrhosis.

\begin{tabular}{|c|c|c|c|}
\hline Characteristics & Normal $(n=49)$ & MHE $(n=42)$ & P-value \\
\hline Disease, B:C:N & $7: 16: 26$ & $5: 12: 25$ & NS \\
\hline Age, years & $60 \pm 14$ & $59 \pm 9$ & NS \\
\hline Gender, f:m & $27: 22$ & $14: 28$ & 0.056 \\
\hline BMI, $\mathrm{kg} / \mathrm{m}^{2}$ & $23.9 \pm 4.0$ & $24.7 \pm 4.9$ & NS \\
\hline CSS & $7.2 \pm 4.7$ & $10.0 \pm 4.7$ & 0.007 \\
\hline ESS & $5.8 \pm 3.9$ & $5.6 \pm 3.5$ & NS \\
\hline PSQI & $5.9 \pm 3.7$ & $7.8 \pm 4.1$ & 0.03 \\
\hline RLS, no:yes & $43: 6$ & $25: 13$ & 0.01 \\
\hline PFN & $36.6 \pm 15.9$ & $33.2 \pm 15.1$ & NS \\
\hline RPN & $77.1 \pm 27.0$ & $28.7 \pm 16.2$ & NS \\
\hline BPN & $50.4 \pm 11.4$ & $44.4 \pm 11.1$ & 0.01 \\
\hline GHN & $40.3 \pm 8.4$ & $38.6 \pm 8.6$ & NS \\
\hline VTN & $44.9 \pm 11.8$ & $41.1 \pm 13.3$ & NS \\
\hline SFN & $44.3 \pm 12.7$ & $39.6 \pm 15.4$ & NS \\
\hline REN & $41.0 \pm 13.9$ & $34.2 \pm 15.3$ & 0.03 \\
\hline MHN & $47.7 \pm 10.7$ & $42.0 \pm 8.7$ & 0.007 \\
\hline PCS & $39.0 \pm 13.3$ & $36.6 \pm 12.9$ & NS \\
\hline MCS & $50.3 \pm 9.6$ & $48.9 \pm 9.5$ & NS \\
\hline RCS & $53.5 \pm 72.6$ & $35.5 \pm 14.8$ & NS \\
\hline MELD & $10.0 \pm 4.3$ & $13.2 \pm 4.3$ & 0.0007 \\
\hline CPS & $6.2 \pm 2.6$ & $8.5 \pm 2.8$ & 0.0001 \\
\hline OHE, no:yes & $47: 2$ & $25: 17$ & 0.0001 \\
\hline BCAA, no:yes & $30: 19$ & $15: 27$ & 0.02 \\
\hline ALB & $3.7 \pm 0.7$ & $3.1 \pm 0.6$ & 0.0001 \\
\hline $\mathrm{TP}$ & $7.26 \pm 0.77$ & $6.89 \pm 0.87$ & 0.03 \\
\hline ALT & $44.3 \pm 43.7$ & $45.8 \pm 38$ & NS \\
\hline $\mathrm{ChE}$ & $215.8 \pm 104.3$ & $130.3 \pm 93$ & 0.0001 \\
\hline $\mathrm{Cr}$ & $0.78 \pm 0.28$ & $1.15 \pm 1.4$ & 0.08 \\
\hline PLT & $13.4 \pm 7.7$ & $8.6 \pm 6.4$ & 0.002 \\
\hline PT-INR & $1.21 \pm 0.36$ & $1.37 \pm 0.35$ & 0.03 \\
\hline $\mathrm{TB}$ & $1.8 \pm 2.5$ & $2.5 \pm 2.3$ & NS \\
\hline HDL & $47.4 \pm 21.6$ & $36.1 \pm 14.9$ & 0.006 \\
\hline LDL & $82.9 \pm 32.2$ & $66.5 \pm 39.3$ & 0.04 \\
\hline $\mathrm{NH}_{3}$ & $46.5 \pm 34.4$ & $82.8 \pm 45.2$ & 0.0001 \\
\hline
\end{tabular}

$\mathrm{B}: \mathrm{C}: \mathrm{N}$, disease caused by hepatitis $\mathrm{B}$ virus:caused by hepatitis $\mathrm{C}$ virus:not caused by hepatitis $B$ virus or hepatitis $C$ virus; MHE, minimal hepatic encephalopathy; f, female; m, male; BMI, body mass index; CSS, cirrhotic symptoms-related score; ESS, Epworth Sleepiness Scale score; PSQI, Pittsburgh Sleep Quality Index; RLS, restless legs syndrome; PFN, physical functioning; BPN, bodily pain; GHN, general health perception; VTN, vitality; SFN, social functioning; REN, emotional health; MHN, mental health; PCS, Physical component summary; MCS, Mental component summary; RCS, Role/Social component summary; MELD, Model for End-stage Liver Disease; CPS, Child-Pugh score; OHE, overt hepatic encephalopathy; BCAA, branched-chain amino acid; ALB, albumin; TP, total protein; ALT, alanine aminotransferase; ChE, cholinesterase; $\mathrm{Cr}$, creatinine; PLT, platelets; PT-INR, prothrombin time-international normalized ratio; TB, total bilirubin; HDL, high-density lipoprotein; LDL, low-density lipoprotein; $\mathrm{NH}_{3}$, ammonia; NS, not statistically significant. 
Table II. Factors associated with minimal hepatic encephalopathy among patients with cirrhosis.

\begin{tabular}{|c|c|c|c|c|c|c|}
\hline \multirow[b]{2}{*}{ Factors } & \multicolumn{3}{|c|}{ Univariate analysis } & \multicolumn{3}{|c|}{ Multivariate analysis } \\
\hline & P-value & OR & $95 \% \mathrm{CI}$ & P-value & OR & $95 \% \mathrm{CI}$ \\
\hline Gender & 0.03 & 2.46 & $1.045-5.763$ & NS & & \\
\hline CSS & 0.01 & 1.14 & $1.029-1.251$ & NS & & \\
\hline PSQI & 0.03 & 1.13 & $1.133-1.006$ & NS & & \\
\hline RLS & 0.01 & 3.73 & $1.258-11.038$ & NS & & \\
\hline BPN & 0.01 & 0.954 & $0.918-0.991$ & NS & & \\
\hline REN & 0.03 & 0.969 & $0.941-0.988$ & NS & & \\
\hline MHN & 0.01 & 0.942 & $0.900-0.986$ & NS & & \\
\hline $\mathrm{OHE}$ & 0.0004 & 15.98 & $3.414-74.805$ & 0.04 & 9.014 & $1.078-75.378$ \\
\hline ALB & 0.0002 & 0.278 & $0.141-0.548$ & 0.06 & 0.339 & $0.107-1.071$ \\
\hline PLT & 0.004 & 0.93 & $0.841-0.969$ & NS & & \\
\hline HDL & 0.009 & 0.967 & 0.944-0.992 & NS & & \\
\hline
\end{tabular}

OR, odds ratio; CI, confidence interval; NS, not statistically significant; CSS, cirrhotic symptoms-related score; PSQI, Pittsburgh Sleep Quality Index; RLS, restless legs syndrome; BPN, body pain; REN, role emotional; MHN, mental health; OHE, overt hepatic encephalopathy; ALB, albumin; PLT, platelets; HDL, high-density lipoprotein.

medication (including BCAA supplements). In addition, the Model for End-stage Liver Disease (MELD) score and CPS were calculated at entry for each patient.

Questionnaires. The CSS questionnaire contained items regarding cirrhotic symptoms, which included hand tremors, appetite loss, foot muscle cramps, fatigue, decreased strength, anxiety, abdominal fullness, abdominal pain, a feeling of low energy, difficulty falling asleep, sleeping poorly and being sleepy during the daytime. An 'impact factor' for each item was calculated, which was defined as the product of the frequency of the item and the mean importance that the patients attributed to the item. The impact factor for each item ranged from 0 to 3 , and the CSS was calculated as the sum of the impact factors (18). The Epworth Sleepiness Scale (ESS) (21) was used to evaluate daytime hypersomnolence; ESS scores range from 0 to 24 , and a score of $\geq 10$ indicates significant daytime hypersomnolence. Sleep quality was evaluated using the Japanese version of the Pittsburgh Sleep Quality Index (PSQI) $(22,23)$. Responses to the PSQI questionnaire were used to generate seven components, which are scored from 0 (normal) to 3 (extremely poor). Health-related quality of life was evaluated using the Japanese SF-36 [version 2; Medical Outcomes Trust (Hanover, NH, USA), Health Lab (Hanover, NH, USA), QualityMetric (Lincoln, RI, USA), and Shunichi Fukuhara (iHope International; Kyoto, Japan)]. This tool contains 1 item that evaluates the perceived change in health status, and the remaining 35 items are used to generate eight subscales of 0-100 that evaluate physical functioning, role limitations due to poor physical health, bodily pain, general health perception, vitality, social functioning, role limitations due to poor emotional health, and role limitations due to poor mental health. All the patients were evaluated for the presence of RLS using a written survey that was developed by the International
Restless Legs Syndrome Research Group in 2003. Patients were diagnosed with RLS if they fulfilled all four criteria and exhibited symptoms of RLS that occurred at least twice per week.

Statistical analysis. All the data were analyzed using Stat Flex software (version 6.0; Artech Co., Ltd., Osaka, Japan) and $\mathrm{P}<0.05$ was considered to indicate a statistically significant difference. Differences in the laboratory data were analyzed using the t-test or $\chi^{2}$ test, as appropriate. A multivariate analysis was performed using binary logistic regression analysis to calculate the odds ratios for development of MHE. Receiver operating characteristic analysis was used to evaluate the association between MHE and CSS with a history of OHE.

\section{Results}

Patients and the NPT. All 91 patients completed the eight tests in the NPT, and abnormal scores were observed in the number connection test A (21 patients), number connection test $\mathrm{B}$ (34 patients), figure position test (79 patients), digit symbol test (60 patients), block design test (29 patients), reaction time test A (65 patients), reaction time test B (62 patients) and reaction time test $C$ (66 patients). In the present study, MHE was defined based on $\geq 2$ abnormal scores in number connection test $\mathrm{A}$, number connection test $\mathrm{B}$, digit symbol test and block design, which identified MHE in 42 of the 91 patients. The clinical characteristics of the normal and MHE groups are shown in Table I. Compared to the normal group, the MHE group exhibited significantly higher values for MELD, CSS, PSQI, RLS, CPS, OHE history, BCAA supplementation, ammonia and prothrombin time-international normalized ratio. Furthermore, compared to the normal group, the MHE group exhibited significantly lower levels of ALB, total 
protein, ChE, PLT, high-density lipoprotein and low-density lipoprotein. Among the SF-36 items, the MHE group exhibited significantly lower bodily pain, emotional health and mental health scores when compared to the normal group. The results of the univariate analyses of the associations between MHE and the characteristics of the patient and scores are listed in Table II. Significant associations were observed between MHE and gender (male), CSS, PSQI, RLS, bodily pain, emotional health, mental health, OHE history, ALB levels, PLT counts and high-density lipoprotein levels. However, only a history of OHE was independently associated with MHE in the multivariate analysis.

Associations for patients without a history of OHE. The 69 patients without a history of OHE (Table III) were also examined and 22 patients were identified who exhibited MHE. Among the patients without a history of OHE, patients with MHE exhibited significantly higher values for CSS, MELD, CPS, prothrombin time-international normalized ratio and ammonia levels when compared to the normal patients. Furthermore, the patients with MHE and no history of OHE exhibited significantly lower levels of ALB, ChE and PLT compared to the normal patients with no history of OHE. However, the multivariate analyses revealed that only CSS was independently associated with MHE among the patients without a history of OHE (Table IV). When the associations between MHE and the CSS items were examined, patients with MHE exhibited significantly higher scores for hand tremors, appetite loss and decreased strength, as well as non-significant increases in the scores for fatigue and anxiety (verses the normal patients) (Table V). However, no significant differences were observed when we compared the scores for muscle cramping, abdominal fullness, abdominal pain, a feeling of low energy and the three sleep-related items.

Predicting MHE. Based on these results, the factors that may predict MHE were evaluated. A history of OHE was the best marker for predicting MHE (odds ratio, 9.014), and CSS was the best marker for predicting MHE in patients without a history of OHE (odds ratio, 1.187). Therefore, a prediction score was developed that combined a history of OHE (no: 0 points, yes: 10 points) and CSS (range, 0-36 points), and evaluated its ability to predict MHE using receiver operating characteristic curve analysis (Fig. 1). The area under the curve was 0.738 and the cut-off value was 8.97 . Based on a cut-off score of 9 points, the prediction score provided a sensitivity of 0.671 and a specificity of 0.333 for MHE.

\section{Discussion}

In the present study, patients were evaluated for signs of MHE, which was indicated by severe liver damage, MELD score, CPS and a history of OHE. In addition, the CSS, PSQI scores and SF-36 items were associated with MHE. The multivariate analyses revealed that a history of $\mathrm{OHE}$ was the best predictor of MHE. However, among patients without a history of OHE, MHE was associated with indicators of severe liver damage, such as CPS, MELD score and platelet counts, and CSS was the best predictor of MHE among those patients. Furthermore, a novel prediction score was developed for MHE using a
Table III. Clinical characteristics of MHE among patients with cirrhosis and no history of overt hepatic encephalopathy.

\begin{tabular}{lccl}
\hline Characteristics & Normal $(\mathrm{n}=47)$ & MHE $(\mathrm{n}=22)$ & P-value \\
\hline CSS & $6.8 \pm 4.4$ & $10.0 \pm 4.7$ & 0.007 \\
MELD & $9.6 \pm 3.3$ & $12.2 \pm 3.7$ & 0.002 \\
CPS & $6.0 \pm 2.5$ & $7.3 \pm 2.5$ & 0.03 \\
ALB & $3.7 \pm 0.7$ & $3.2 \pm 0.6$ & 0.003 \\
ChE & $220.1 \pm 103.2$ & $149.5 \pm 105.8$ & 0.007 \\
PLT & $13.6 \pm 7.8$ & $9.2 \pm 7.4$ & 0.02 \\
PTI-NR & $1.12 \pm 0.17$ & $1.35 \pm 0.43$ & 0.01 \\
$\mathrm{NH}_{3}$ & $45.2 \pm 34.6$ & $75.1 \pm 45.5$ & 0.004 \\
\hline
\end{tabular}

MHE, minimal hepatic encephalopathy; CSS, cirrhotic symptoms-related score; MELD, Model for End-stage Liver Disease; CPS, Child-Pugh score; ALB, albumin; ChE, cholinesterase; PLT, platelets; PT-INR, prothrombin time-international normalized ratio; $\mathrm{NH}_{3}$, ammonia.

history of OHE and CSS, which provided an area under the curve of 0.738 and a sensitivity of 0.671 .

Similar to these findings, it has been reported that patients with MHE exhibit severe liver damage (as indicated by a high MELD score) $(10,24,25)$ and high CPS (24). However, the previous studies did not include patients with a history of OHE $(23,24)$. Moreover, OHE and MHE are not fully treated via liver transplantation $(19,26)$, and patients with a history of OHE may experience impaired cognitive function, even subsequent to resolving the OHE (27). Therefore, patients with resolved $\mathrm{OHE}$ may be at risk for developing MHE, regardless of the extent of any liver damage. Furthermore, MHE is a risk factor for $\mathrm{OHE}$ (9), and resolution of $\mathrm{OHE}$ is a risk factor for MHE, which highlights the importance of accurately determining whether patients with cirrhosis have a history of OHE. The present findings confirm this concept, as a history of $\mathrm{OHE}$ was the best predictor of MHE among all the variables that were evaluated.

It is well known that BCAA affects MHE and OHE (16), there were a few patients with a history of OHE who had already treated with BCAA in this study. Therefore, the MHE group had a significantly higher number of patients who were treated with BCAA supplements compared to the normal group. Thus, there was a possibility of the effects that BCAA supplements improve OHE to MHE.

Patients without a history of OHE were also evaluated, and as speculated, their sleep disturbances may be associated with MHE. Notably, low PSQI scores and frequent RLS were associated with MHE among patients with a history of OHE, although these associations were not observed among patients without a history of OHE. Furthermore, CSS was the best predictor of MHE among the patients without a history of OHE. In this context, CSS is calculated using items from a cirrhosis symptom questionnaire that was developed in our previous study, and three of the 12 items in this tool are associated with sleep disturbances. Moreover, our previous study reported that CSS, excluding the scores for the three 
Table IV. Factors associated with minimal hepatic encephalopathy among patients with cirrhosis and no history of overt hepatic encephalopathy.

\begin{tabular}{|c|c|c|c|c|c|c|}
\hline \multirow[b]{2}{*}{ Factors } & \multicolumn{3}{|c|}{ Univariate analysis } & \multicolumn{3}{|c|}{ Multivariate analysis } \\
\hline & P-value & OR & $95 \% \mathrm{CI}$ & P-value & OR & $95 \% \mathrm{CI}$ \\
\hline CSS & 0.01 & 1.17 & $1.034-1.313$ & 0.02 & 1.187 & $1.023-1.379$ \\
\hline ALB & 0.005 & 0.35 & $0.167-0.782$ & NS & & \\
\hline ChE & 0.01 & 0.993 & 0.993-0.998 & NS & & \\
\hline $\mathrm{NH}_{3}$ & 0.007 & 1.019 & $1.005-1.033$ & NS & & \\
\hline PLT & 0.03 & 0.918 & 0.849-0.991 & NS & & \\
\hline PT-INR & 0.02 & 27.19 & $1.531-482.865$ & NS & & \\
\hline
\end{tabular}

OR, odds ratio; CI, confidence interval; NS, not statistically significant; CSS, cirrhotic symptoms-related score; ALB, albumin; ChE, cholinesterase; $\mathrm{NH}_{3}$, ammonia; PLT, platelets; PT-INR, prothrombin time-international normalized ratio.

Table V. Comparing the cirrhosis-related symptom scores among patients with and without MHE.

\begin{tabular}{lccc}
\hline Questionnaire items & MHE & Normal & P-value \\
\hline Hand tremors & 0.524 & 0.196 & 0.01 \\
Appetite loss & 0.957 & 0.413 & 0.004 \\
Muscle cramping & 1.174 & 0.957 & $\mathrm{NS}$ \\
Fatigue & 1.478 & 1.064 & 0.09 \\
Decreased strength & 1.957 & 1.468 & 0.02 \\
Anxiety & 1.174 & 0.787 & 0.06 \\
Abdominal fullness & 0.957 & 0.638 & $\mathrm{NS}$ \\
Abdominal pain & 0.391 & 0.340 & $\mathrm{NS}$ \\
Feeling of low energy & 1.391 & 1.109 & $\mathrm{NS}$ \\
Difficulty falling asleep & 1.391 & 1.128 & $\mathrm{NS}$ \\
Poor sleep quality & 1.478 & 1.289 & $\mathrm{NS}$ \\
Sleepy during the daytime & 1.318 & 1.340 & $\mathrm{NS}$ \\
\hline
\end{tabular}

MHE, minimal hepatic encephalopathy; NS, not statistically significant.

sleep disturbance-related items, is associated with liver damage (18). In the present study, three CSS items (hand tremors, appetite loss and decreased strength) were significantly associated with MHE, and these associations validate the previously reported associations between MHE and the following responses: 'I do not maintain balance', 'I act irritable or impatient with myself', 'I am not doing any of my usual physical recreations or activity' and 'I am eating much less than usual' (25). Another study has also reported that worry was strongly associated with MHE in a questionnaire regarding chronic liver disease (24). Although the majority of the patients with MHE experience symptoms that contribute to cognitive impairment, their other limitations (such as hand tremors, appetite loss and decreased strength) may also reflect restrictions in their activities of daily living. By contrast, MHE was not associated with difficulty in falling asleep,

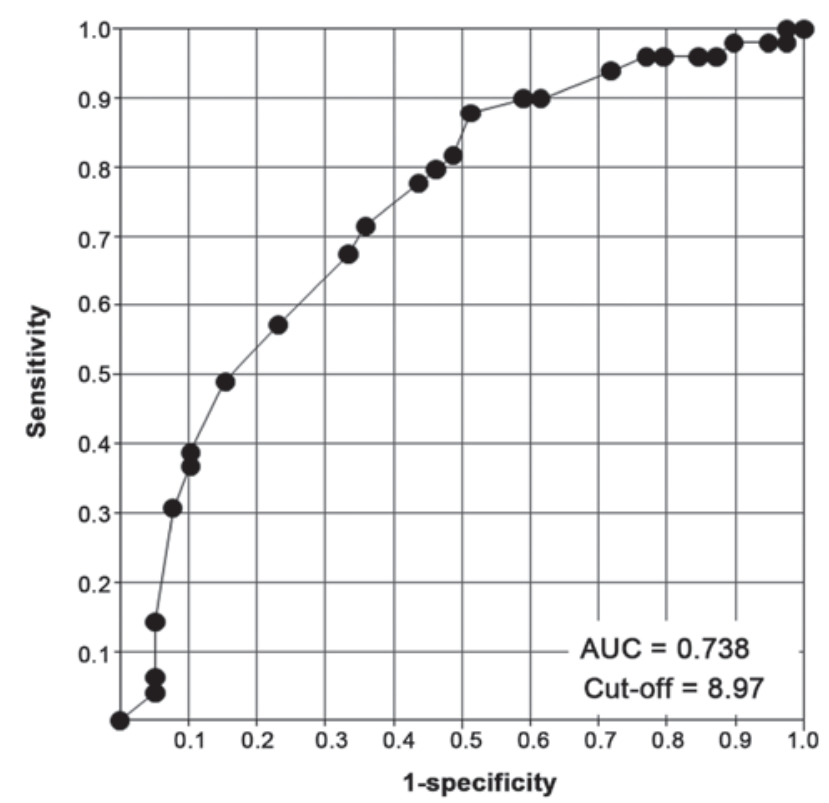

Figure 1. Receiver operating characteristic curve for minimal hepatic encephalopathy in patients with cirrhosis. The area under the curve (AUC) and optimal cut-off value are indicated.

sleeping poorly and being sleepy during the daytime, which would indicate that patients with MHE, but not OHE, do not experience sleep disturbances.

In conclusion, the present findings indicate that a history of OHE was the best predictor of MHE, and CSS was the best predictor of MHE among patients without a history of OHE. Therefore, a prediction score was developed using a history of OHE and CSS, and this score appears to be effective in screening for MHE among patients with cirrhosis. However, this score is not highly specific for MHE, and additional parameters are required to develop a more sensitive screening tool. As patients with cirrhosis have multiple symptoms, which may be associated with cognitive impairment (28), the incorporation of cognitive impairment symptoms into the present prediction score may increase its sensitivity for MHE. 


\section{References}

1. Romero-Gómez M, Montagnese S and Jalan R: Hepatic encephalopathy in patients with acute decompensation of cirrhosis and acute-on-chronic liver failure. J Hepatol 62: 437-447, 2015.

2. Ferenci P, Lockwood A, Mullen K, Tarter R, Weissenborn K and Blei AT: Hepatic encephalopathy - definition, nomenclature, diagnosis, and quantification: Final report of the working party at the 11th World Congresses of Gastroenterology, Vienna, 1998. Hepatology 35: 716-721, 2002.

3. Kato A, Watanabe Y, Sawara K and Suzuki K: Diagnosis of sub-clinical hepatic encephalopathy by Neuropsychological Tests (NP-tests). Hepatol Res 38 (Suppl 1): S122-S127, 2008.

4. Qi R, Zhang LJ, Luo S, Ke J, Kong X, Xu Q, Liu C, Lu H and Lu GM: Default mode network functional connectivity: A promising biomarker for diagnosing minimal hepatic encephalopathy: CONSORT-compliant article. Medicine (Baltimore) 93: e227, 2014

5. Cona G, Montagnese S, Bisiacchi PS, Gatta A, Cillo U, Angeli P, Amodio P and Schiff S: Early markers of neural dysfunction and compensation: A model from minimal hepatic encephalopathy. Clin Neurophysiol 125: 1138-1144, 2014.

6. Kircheis G, Hilger N and Häussinger D: Value of critical flicker frequency and psychometric hepatic encephalopathy score in diagnosis of low-grade hepatic encephalopathy. Gastroenterology 146: 961-969, 2014.

7. Montoliu C, Cauli O, Urios A, ElMlili N, Serra MA, Giner-Duran R, González-Lopez O, Del Olmo JA, Wassel A, Rodrigo JM, et al: 3-nitro-tyrosine as a peripheral biomarker of minimal hepatic encephalopathy in patients with liver cirrhosis. Am J Gastroenterol 106: 1629-1637, 2011.

8. Montoliu C, Piedrafita B, Serra MA, del Olmo JA, Urios A, Rodrigo JM and Felipo V: IL-6 and IL-18 in blood may discriminate cirrhotic patients with and without minimal hepatic encephalopathy. J Clin Gastroenterol 43: 272-279, 2009.

9. Riggio O, Amodio P, Farcomeni A, Merli M, Nardelli S, Pasquale C, Pentassuglio I, Gioia S, Onori E, Piazza N, et al: A Model for Predicting Development of Overt Hepatic Encephalopathy in Patients With Cirrhosis. Clin Gastroenterol Hepatol 13: 1346-1352, 2015.

10. Ampuero J, Simón M, Montoliú C, Jover R, Serra MÁ, Córdoba J and Romero-Gómez M: Minimal hepatic encephalopathy and critical flicker frequency are associated with survival of patients with cirrhosis. Gastroenterology 149: 1483-1489, 2015.

11. Bajaj JS, Saeian K, Schubert CM, Hafeezullah M, Franco J, Varma RR, Gibson DP, Hoffmann RG, Stravitz RT, Heuman DM, et al: Minimal hepatic encephalopathy is associated with motor vehicle crashes: The reality beyond the driving test. Hepatology 50: 1175-1183, 2009.

12. Bajaj JS, Pinkerton SD, Sanyal AJ and Heuman DM: Diagnosis and treatment of minimal hepatic encephalopathy to prevent motor vehicle accidents: A cost-effectiveness analysis. Hepatology 55: 1164-1171, 2012.

13. Kawaguchi T, Taniguchi E and Sata M: Motor vehicle accidents: How should cirrhotic patients be managed? World J Gastroenterol 18: 2597-2599, 2012.

14. Román E, Córdoba J, Torrens M, Torras X, Villanueva C, Vargas V, Guarner C and Soriano G: Minimal hepatic encephalopathy is associated with falls. Am J Gastroenterol 106: 476-482, 2011.
15. Bianchi G, Giovagnoli M, Sasdelli AS and Marchesini G: Hepatic encephalopathy and health-related quality of life. Clin Liver Dis 16: 159-170, 2012.

16. Kato A, Tanaka H, Kawaguchi T, Kanazawa H, Iwasa M, Sakaida I, Moriwaki H, Murawaki Y, Suzuki K and Okita K: Nutritional management contributes to improvement in minimal hepatic encephalopathy and quality of life in patients with liver cirrhosis: A preliminary, prospective, open-label study. Hepatol Res 43: 452-458, 2013

17. Matsuzaki T, Ichikawa T, Kondo H, Taura N, Miyaaki H, Isomoto $\mathrm{H}$, Takeshima $\mathrm{F}$ and Nakao K: Prevalence of restless legs syndrome in Japanese patients with chronic liver disease. Hepatol Res 42: 1221-1226, 2012.

18. Ichikawa $T$, Naota $T$, Miyaaki $H$, Miuma S, Isomoto $H$, Takeshima F and Nakao K: Effect of an oral branched chain amino acid-enriched snack in cirrhotic patients with sleep disturbance. Hepatol Res 40: 971-978, 2010.

19. Akahoshi M, Ichikawa T, Taura N, Miyaaki H, Yamaguchi T, Yoshimura E, Takahara I, Soyama A, Takatsuki M, Kondo H, et al: Sleep disturbances and quality of life in patients after living donor liver transplantation. Transplant Proc 46: 3515-3522, 2014.

20. Fukuhara S and Suzukamo Y: Manual of the SF-36v2 Japanese Version. Institute for Health Outcomes and Process Evaluation Research, Kyoto, Japan, 2004.

21. Johns MW: A new method for measuring daytime sleepiness: The Epworth sleepiness scale. Sleep 14: 540-545, 1991.

22. Doi Y, Minowa M, Uchiyama M, Okawa M, Kim K, Shibui K and Kamei Y: Psychometric assessment of subjective sleep quality using the Japanese version of the Pittsburgh Sleep Quality Index (PSQI-J) in psychiatric disordered and control subjects. Psychiatry Res 97: 165-172, 2000.

23. Buysse DJ, Reynolds CF III, Monk TH, Berman SR and Kupfer DJ: The Pittsburgh Sleep Quality Index: A new instrument for psychiatric practice and research. Psychiatry Res 28: 193-213, 1989.

24. Hirano H, Saito M, Yano Y, Momose K, Yoshida M, Tanaka A and Azuma T: Chronic liver disease questionnaire would be a primary screening tool of neuropsychiatric test detecting minimal hepatic encephalopathy of cirrhotic patients. Hepatol Res 45: 994-1003, 2015.

25. Nabi E, Thacker LR, Wade JB, Sterling RK, Stravitz RT, Fuchs M, Heuman DM, Bouneva I, Sanyal AJ, Siddiqui MS, et al: Diagnosis of covert hepatic encephalopathy without specialized tests. Clin Gastroenterol Hepatol 12: 1384-1389.e2, 2014.

26. Lin WC, Chou KH, Chen CL, Chen HL, Lu CH, Li SH, Huang CC, Lin CP and Cheng YF: Longitudinal brain white matter alterations in minimal hepatic encephalopathy before and after liver transplantation. PLoS One 9: e105887, 2014.

27. Umapathy S, Dhiman RK, Grover S, Duseja A and Chawla YK: Persistence of cognitive impairment after resolution of overt hepatic encephalopathy. Am J Gastroenterol 109: 1011-1019, 2014.

28. Córdoba J: New assessment of hepatic encephalopathy. J Hepatol 54: 1030-1040, 2011. 